\title{
USO DE PARAMETRIZAÇÃO DIGITAL NO DESENVOLVIMENTO DE ASSENTOS PERSONALIZADOS PARA CADEIRAS DE RODAS
}

\section{USE OF DIGITAL PARAMETERIZATION FOR THE DEVELOPMENT OF CUSTOM WHEELCHAIR SEATS}

\author{
Catherine Teixeira Marcon ${ }^{7}$ \\ Bruna da Silveira Suris ${ }^{2}$ \\ Underléia Miotto Bruscato ${ }^{3}$ \\ Mariana Pohlmann de Oliveira ${ }^{4}$ \\ Fabio Pinto da Silva ${ }^{5}$
}




\section{Resumo}

O assento personalizado é um recurso de Tecnologia Assistiva destinado a melhorar a adequação postural dos usuários de cadeira de rodas. O objetivo deste estudo é otimizar a fabricação de assentos com baixo custo por meio do design paramétrico. O método proposto é baseado na identificação e parametrização digital da anatomia dos usuários impressa em uma almofada moldável a vácuo. Após a aquisição manual de coordenadas, o algoritmo criado reproduz digitalmente a forma desejada, a qual, possibilitaria a usinagem de uma espuma para o assento. Como validação da proposta, realizou-se a comparação entre a forma reconstruída pelo algoritmo e a forma original da almofada digitalizada com um escâner 3D.

Palavras-chave: Tecnologia Assistiva; Digitalização 3D; Design Paramétrico; Fabricação Digital; Grasshopper.

\section{Abstract}

A custom wheelchair seat is an Assistive Technology feature designed to improve users' postural adaptation. The aim of this study is to optimize seats manufacture at low cost through parametric design. The proposed method is based on identification and digital parameterization of the users' shape printed on a vacuum moldable positioning pillow. After manual acquisition of coordinates, the created algorithm digitally reproduces the desired shape that would allow a seat cushion machining. As validation of the proposal, a comparison was made between the algorithm reconstructed shape and the pillow original shape digitalized with a 3D scanner.

Key-words: Assistive Technology; 3D Scanning; Parametric Design; Digital Fabrication; Grasshopper.

${ }^{1}$ caatherine1@gmail.com

2 bruna_suris@hotmail.com

3 arq.leiab@gmail.com

${ }^{4}$ marianapohlmann@gmail.com

${ }^{5}$ fabio.silva@ufrgs.br 


\section{INTRODUÇÃO}

O design paramétrico está inserido no desenvolvimento e na evolução das técnicas de manufatura, o que tem permitido que o designer atue sob novas perspectivas projetuais (TEDESCHI, 2014; DEMARCHI et al., 2017). A parametrização digital, então, permite que sejam alterados dados variados, ou seja, parâmetros pré-definidos e é responsável por gerar variações de um mesmo projeto, o que favorece o desenvolvimento de produtos personalizados para o usuário. Nesse sentido, o presente trabalho foca na utilização da parametrização digital como uma ferramenta para o desenvolvimento de produtos de Tecnologia Assistiva (TA), mais especificamente, de assentos personalizados para cadeiras de rodas.

A Tecnologia Assistiva é definida como uma área do conhecimento com característica interdisciplinar, que envolve desde produtos, recursos e serviços, práticas e estratégias que ampliem as habilidades funcionais, assim como, promovam a autonomia, independência e inclusão social de pessoas com deficiência (BRASIL, 2009). Ressalta-se a importância de seu desenvolvimento visando à melhoria da qualidade de vida das pessoas com deficiência ou mobilidade reduzida.

A Cadeira de Rodas (CR) é um dos equipamentos de TA mais utilizados para proporcionar a mobilidade pessoal e com isso, auxiliar as pessoas com deficiência a adquirirem participação na sociedade (OMS, 2014). No âmbito do Programa Technology Initiative for Disabled and Elderly People - TIDE, segundo o modelo de classificação Horizontal European Activities in Rehabilitation Technology - HEART, o equipamento faz parte de uma das três principais áreas de TA, sendo elas: componentes técnicos, componentes humanos e componentes sociais. Sua aquisição exige uma avaliação personalizada, mediante as necessidades e características de cada usuário, para, assim, ser definida a CR mais adequada para cada caso (TEIXEIRA et al., 2003; BRASIL, 2009). Considerando as diferenças antropométricas de cada sujeito, deve-se considerar que toda pessoa apresenta uma postura corporal diferente, seja por questões como obesidade, fraqueza óssea ou hipotonia muscular, podendo vir a desenvolver a necessidade do auxílio de dispositivos personalizados (PONTES, 2017).

Nesse contexto, um conceito importante é o de Adequação Postural (AP), definida por Bersch (2018) como "adaptações para cadeira de rodas ou outro sistema de sentar visando o conforto e distribuição adequada da pressão na superfície da pele (almofadas especiais, assentos e encostos anatômicos) [...]". A AP tem um importante papel de proporcionar ao usuário o controle postural necessário para a realização das atividades de vida diária e interação com o ambiente.

Sabe-se que o uso de cadeiras de rodas sem adaptações corretas pode causar danos ao usuário, prejudicando funções básicas e alterações no sistema circulatório, assim como, a má postura pode vir a refletir no surgimento de dores e restrições de movimentos (PONTES, 2017). Além disso, em casos mais críticos, o uso de dispositivos não adaptados acaba prejudicando a postura do usuário de $\mathrm{CR}$, podendo ser um dos fatores determinantes para as úlceras de pressão, por exemplo (ENGSTRÖM, 2002; TEIXEIRA et al., 2003; SILVA, 2011).

Porém, é preciso ressaltar que independente de todas as modificações, conforme as variações e requerimentos de cada usuário, o conforto gerado pelo assento deverá 
ser uma constante independente. Ademais, sobre a influência que os custos da produção de um produto personalizado têm sobre estes dispositivos de TA:

\begin{abstract}
É importante que se perceba que de nada adianta o cumprimento de todas as etapas da prestação de serviços de Tecnologia Assistiva - incluindo pesquisa e desenvolvimento - se o acesso à ferramenta adequada esbarra em restrições orçamentárias, ou se a viabilidade mercadológica de um produto torna necessário o empobrecimento do seu projeto, como, por exemplo, a redução da variabilidade e da ajustabilidade dos equipamentos (BERSCH et al., 2008, p.18).
\end{abstract}

A união entre a personalização e o baixo custo são de interesse para solucionar esses problemas existentes referentes à TA. Contudo, a questão é que "a realidade do processo de fabricação de produtos de TA é a massificação e, em busca de uma produção de menor custo, a indústria optou por padronizar processos, dimensões e densidades" (DEMARCHI et al., 2017, p. 2). Isso acaba ignorando a necessidade de produtos personalizados adequadamente à diversidade antropométrica e funcional das pessoas com deficiência.

Frente aos levantamentos aqui apontados, percebe-se que há uma demanda referente ao desenvolvimento de recursos de Tecnologia Assistiva voltados para a adequação postural em cadeiras de rodas e a personalização destes, enfatizando o baixo custo envolvido. Porém, sabe-se que no cenário atual a flexibilização de produtos personalizados, ainda pode ser restrita para grande parte da população brasileira, em função dos altos custos de produção. De acordo com Fredriksson e Gade (2005) para reduzir os custos finais do produto, é indispensável reorganizar as estruturas das atividades produtivas, o que acarretará no aumento da flexibilidade do processo. Diante do exposto, é preciso focar em sistemas produtivos personalizados que trazem agilidade ao projeto, como processos automatizados.

Assim, com o objetivo de otimizar a fabricação de assentos personalizados para CR sem elevar custos, este estudo investiga a aplicabilidade do design paramétrico como ferramenta. A partir da parametrização, integrada com recursos de fabricação digital, busca-se desenvolver assentos personalizados, os quais possam prover conforto, estabilidade e adequação postural de pessoas com deficiência.

\title{
2 ADEQUAÇÃO POSTURAL EM CADEIRA DE RODAS
}

Moraes (2009) aponta que existem basicamente dois componentes integrados em uma CR, a saber: a) componente veicular - sendo responsável por algumas características técnicas como estabilidade, autonomia, manobrabilidade, raio de giro, amortecimento de vibrações e eficiência de propulsão; b) componente de adequação postural - voltado para o conforto, facilidade de desenvolvimento das atividades motoras, estabilização da postura da cabeça, prevenção e correção de possíveis deformidades e facilidade de cuidados por terceiros. Nesta mesma linha, Teixeira et al. (2003) determinam algumas características que a cadeira de rodas deve possuir:

- Ser confortável: a cadeira de rodas é a extensão de um corpo incapacitado, e a pessoa passará grande parte de seu tempo nesse equipamento, geralmente na mesma posição. 
- Aliviar a pressão: a distribuição do peso corporal tem de ser uniforme, favorecendo, assim, o alívio dos pontos de pressão. (...)

- Incrementar a função: o sistema tem de possibilitar ao indivíduo a otimização e a maximização das funções motoras (...), fisiológicas (...), entre outras.

- Proporcionar suporte corporal: o sistema deve oferecer suporte corporal adequado para a pessoa com deficiência de acordo com as suas necessidades, (...)

- Permitir alterações e ajustes: o sistema deve ser planejado de forma que atenda as necessidades atuais (...), mas deve permitir modificações (...) (Teixeira et al., 2003, p. 176 e 177)

Filho (2003) discorre sobre as condições de conforto, colocando-as como circunstância de comodidade e bem-estar. Então, é necessário destacar que os produtos que contrariam as considerações sobre o conforto podem ocasionar fadiga, doenças futuras, lesões na pele, dentre outros malefícios ao organismo do usuário (ENGSTRÖM, 2002; KRÜGER e FERREIRA, 2013).

A qualidade de vida dos sujeitos que utilizam a CR, dos quais a maioria passa a maior parte do dia sentado na mesma posição, é dependente diretamente do conforto que esta proporciona (MORAES, 2009; RENNER et al. 2018). O que leva a considerar que, segundo a Organização Mundial da Saúde, "todos os usuários de cadeira de rodas devem estar confortáveis em sua cadeira de rodas e uma boa almofada ajuda a se sentarem eretos com facilidade e conforto" (OMS, 2014). Sentar-se ereto contribui para a distribuição do peso do usuário proporcionalmente na superfície do assento e também auxilia no seu alinhamento. Há uma grande variabilidade de parâmetros anatômicos que precisam ser considerados para a confecção de assentos de CR (TEIXEIRA et al. 2003; SILVA, 2011; BERSCH, 2018).

Crytzer et al. (2016) salientam que a posição sentada das pessoas, de forma geral, é muito variada e quando estas envolvem indivíduos usuários de CR, algumas deformidades na coluna vertebral resultam no desenvolvimento de contornos posteriores que são demasiadamente diferentes de uma forma "padrão". Bersch (2018) discorre sobre a questão da AP, a qual, segundo a autora, refere-se à seleção de recursos que possam garantir posturas estáveis e com boa distribuição do peso corporal, enfatizando que os usuários de CR devem ser beneficiados por sistemas de assentos desenvolvidos com base nas suas medidas e no seu peso, considerando suas limitações e capacidades. Além disso, uma almofada adequada ajuda a aliviar a pressão e traz suporte para que o usuário não escorregue (ENGSTRÖM, 2002).

Porém, existem diferentes tipos de almofadas, as quais devem ser definidas, conforme sua função principal e necessidades específicas dos usuários (OMS, 2014). Teixeira et al. (2003) apontam alguns, por exemplo, sujeitos acometidos pela mielomeningocele necessitam de assentos rígidos, planos e com variação de densidades ao longo do mesmo. Já para pessoas com paralisia cerebral é indicada a base rígida, porém, anatômica e também outro modelo que proporciona maior contato corporal, o qual é gerado a partir da digitalização de uma almofada simuladora (TEIXEIRA et al., 2003). 
Nesse contexto, o estudo aqui apresentado aponta para a personalização de assentos baseados na anatomia do usuário. Para tanto, observa-se a tecnologia de digitalização tridimensional como uma interessante alternativa, mas, que pode acarretar em um maior custo de fabricação e dificuldade de acesso.

\section{DIGITALIZAÇÃO TRIDIMENSIONAL E DESIGN PARAMÉTRICO}

De acordo com Silva (2006), utiliza-se o método de digitalização tridimensional com o objetivo de captar dados precisos advindos de objetos físicos, para obter resultados quanto a superfícies, texturas e até objetos inteiros, com o auxílio de ferramentas computacionais. Na digitalização 3D adquirem-se coordenadas $x, y$ e z, as quais, após processamento, tornam possíveis manipulações por sistemas CAD/CAM (Projeto Auxiliado por Computador / Manufatura Auxiliada por Computador). Com o modelo digital pode-se realizar avaliações, construir moldes, analisar superfícies e, a partir dos resultados, realizar o desenvolvimento de produtos adequados às necessidades do usuário. Entretanto, a maioria desses sistemas possui um alto custo, especialmente na realidade do Brasil, o que acaba dificultando o seu uso em projetos com verba reduzida (SILVA, 2011).

Costa et al. (2015) salientam sobre os avanços da tecnologia e o fato de abrirem as portas para as pesquisas referentes à obtenção de dados antropométricos, por meio de digitalizadores 3D. Nesse contexto, Silva (2011) propõe um método de fabricação de assentos e encostos de CR fabricados sob medida, o processo ocorre a partir da digitalização 3D e culmina com a usinagem de uma espuma de poliuretano flexível. Outros pesquisadores também exploram a digitalização tridimensional para fabricação de recursos de TA, como Moraes (2009), Beretta (2011), Fernandes et al. (2014) e Crytzer et al. (2016).

Porém, há um problema em manter os benefícios da digitalização 3D aplicada à adequação postural, considerando o estado da tecnologia x custo. Dado o alto valor dos mais atuais equipamentos ópticos para digitalização 3D, uma alternativa de baixo custo para a aquisição da anatomia do usuário pode ser a captura manual com contato (com o uso de instrumentos de medição convencionais). Assim, a inserção do design paramétrico para modelar as coordenadas surge como uma possibilidade.

O desenho paramétrico vem se mostrando eficaz no que diz respeito à flexibilidade e agilidade do processo produtivo. Nesse conceito, um produto é projetado a partir de um conjunto de parâmetros, relacionados ao uso de ferramentas digitais, em que computadores são programados com algoritmos para executar tarefas necessárias à realização de um objetivo. Tudo isso com o intuito de aumentar a efetividade de um processo (OXMAN e OXMAN, 2014; TEDESCHI, 2014).

Tratando-se de parâmetros, eles podem simplesmente ter efeito sobre a quantidade de um material, ou se tornarem mais avançados, como na verificação de esforços de uma estrutura. No contexto do design e da arquitetura, por exemplo, os parâmetros codificam e quantificam as opções e restrições dentro de um sistema em que, o design do objeto é substituído pelo design do processo que gera o objeto (OXMAN e OXMAN, 2014; TEDESCHI, 2014).

Dentre as vantagens da parametrização, tem-se a possibilidade de criação de formas complexas, mais orgânicas, bem como de aproveitamento dos materiais e de 
otimização e adaptação a materiais diversos (OXMAN e OXMAN, 2014; TEDESCHI, 2014; DEMARCHI et al., 2017). Em se tratando de formas complexas, segundo Pires et al. (2017), os sistemas digitais paramétricos permitem o aumento do controle sobre a variedade de alternativas de um mesmo projeto, bem como, soluções customizadas. Assim, há a vantagem de facilitar o processo de criação e desenvolvimento de projetos, o que normalmente exige inúmeras modificações e ocasiona a frustração dos profissionais envolvidos por conta do retrabalho (FLORIO, 2012).

Oxman e Oxman (2014), Tedeschi (2014) e Demarchi et al. (2017) exemplificam programas gráficos computacionais como o software Rhinoceros, e seu editor visual de algoritmos Grasshopper, que facilitam a parametrização para usuários que possuam pouco ou nenhum conhecimento em linguagens de programação. Esses softwares podem ser empregados no desenvolvimento de projetos de assentos personalizados para cadeiras de rodas. Nesse sentido, o presente estudo propõe a aquisição de dados antropométricos manualmente e a modelagem paramétrica, para geração de um arquivo virtual que, posteriormente, pode ser utilizado para a fabricação digital de moldes ou assentos personalizados.

\section{MATERIAIS E MÉTODOS}

Para alcançar o objetivo proposto, estabeleceu-se a seguinte metodologia: uma pesquisa de natureza aplicada, de abordagem qualitativa, de modalidade descritiva e procedimento experimental. Dessa forma, este capítulo é destinado à descrição dos materiais e métodos adotados. Frequentemente empregada nas ciências tecnológicas, na pesquisa experimental, é determinado um objeto de estudo, são definidas as variáveis que podem influenciá-lo e é selecionada a maneira de observação e controle dos seus efeitos. Diante disso, é necessário um espaço controlado e instrumentos de pesquisa apropriados, para verificação dos resultados encontrados (GIL, 2008; PRODANOV, 2013).

Para iniciar o estudo, foi necessário elaborar um algoritmo para modelagem paramétrica. $O$ algoritmo proposto recebe as coordenadas de um assento pré-moldado a vácuo com a anatomia do usuário para convertê-las em um modelo 3D. Assim, foi verificada a necessidade de um gabarito para auxiliar os procedimentos. Inicialmente, a moldagem do usuário deve ocorrer dentro de uma caixa de base quadrada e aberta em uma das laterais (na direção das pernas do usuário), a qual limita as dimensões externas do assento. Posteriormente, as medições devem ser realizadas dentro de uma grade com igual espaçamento em x e y (considerando z, a altura do assento). Por fim, as coordenadas medidas manualmente devem ser introduzidas no algoritmo que gera o modelo 3D parametrizado. Para verificar a qualidade do resultado obtido, o molde original do usuário foi digitalizado com um escâner 3D para comparação entre os modelos. Todos os equipamentos utilizados ao longo do estudo pertencem ao Laboratório de Design e Seleção de Materiais (LDSM), da Universidade Federal do Rio Grande do Sul (UFRGS).

Ainda no que se refere à metodologia da pesquisa, a seguir são apresentados detalhadamente cada um dos procedimentos utilizados. Sendo eles: desenvolvimento do gabarito, desenvolvimento do algoritmo paramétrico, moldagem e execução e, por fim, análise do modelo paramétrico. 


\subsection{Desenvolvimento do gabarito}

Com a necessidade de se desenvolver um gabarito, projetou-se uma caixa vazada em sua porção frontal com o tampo removível. As peças que a compõem foram desenhadas no software Rhinoceros e cortadas a laser em MDF (Medium Density Fiberboard), com $6 \mathrm{~mm}$ de espessura. Conforme observa-se na Figura 1, o gabarito é composto por um paralelepípedo vazado (faces superior e frontal). As proporções consideradas foram baseadas em uma CR comum. Os limites do assento da CR são de $400 \times 400 \mathrm{~mm}$ (interior do gabarito), de acordo com o padrão definido pela ABNT NBR ISO 7176-7:2009 que é descrita e analisada no Programa de Análise de Produtos do INMETRO (BRASIL, 2013).

Figura 1 - Gabarito para moldagem do usuário com chapa superior para realização de medições

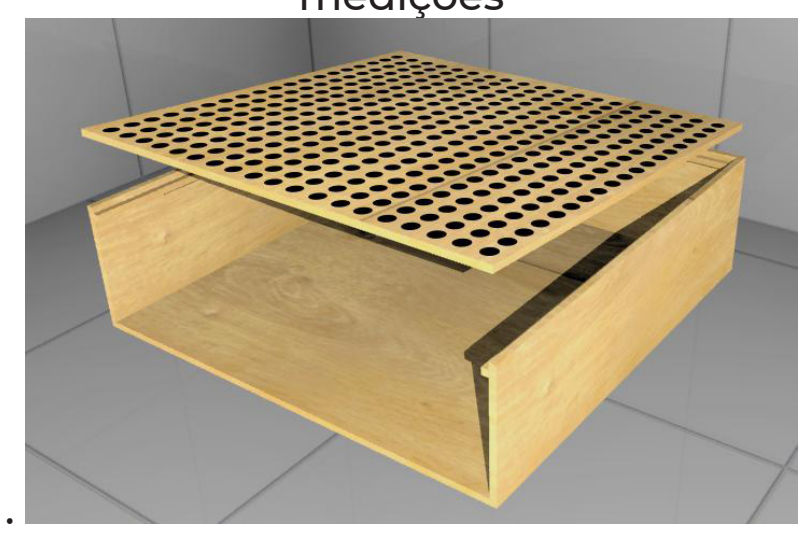

Fonte: Elaborado pelos autores, 2019.

No interior da caixa deve ser acomodada uma almofada moldável de posicionamento à vácuo (Figura 2A), onde o sujeito senta-se, moldando sua forma anatômica. A altura da caixa foi definida em $100 \mathrm{~mm}$, que seria o limite de profundidade do assento, sendo suficiente para a espessura da almofada. Após a moldagem, insere-se o tampo para realizar as medições.

Figura 2 - Gabarito: A) com almofada moldável de posicionamento à vácuo; B) com tampo de referência para medição.
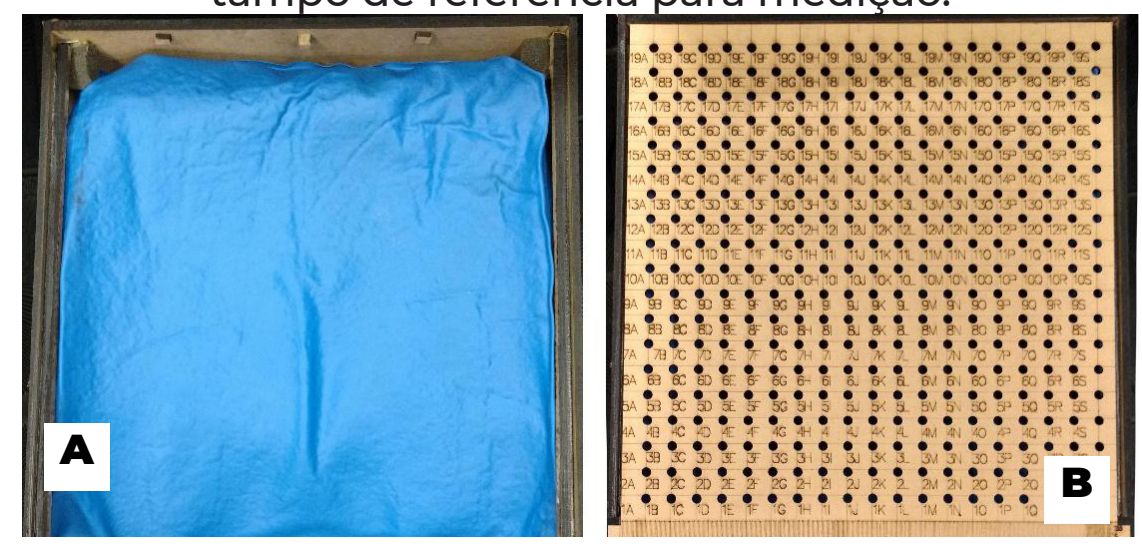

Fonte: Elaborado pelos autores, 2019. 
Para a referência espacial, necessária ao algoritmo desenvolvido, foi tomado um plano de referência (xy), que é o tampo removível do gabarito. Assim, o ponto zero do eixo z fica determinado pela face superior do tampo, o qual terá uma variação interna de até $100 \mathrm{~mm}$ (distância até a base da caixa). O tampo possui 361 furos (grade de 19 x 19 furos), igualmente espaçados em $20 \mathrm{~mm}$ para cada eixo (x e y). Os furos são pontos de referência para as medições, que podem ser realizadas inserindo a haste de profundidade de um paquímetro. Embora, com a furação proposta, seja viável realizar medições com resolução de até $20 \mathrm{~mm}$, tecnicamente, seria possível obter resoluções mais grosseiras que tornam a medição mais rápida, bastando utilizar menos pontos de medição. Para facilitar a referência, os furos receberam legendas em colunas $(x)$ e linhas (y), respectivamente, identificados com letras (do A ao S) e números (do 1 ao 19). O tampo gravado a laser pode ser observado na Figura 2B.

\subsection{Desenvolvimento do algoritmo paramétrico}

O algoritmo proposto foi desenvolvido no editor visual Grasshopper, integrado ao software de modelagem Rhinoceros. O ponto de partida foi uma lista de números, que pode ser tabulada em editores de planilha convencionais, como o Microsoft Excel, ou mesmo, em editores de texto. Os dados foram importados pelo algoritmo e utilizados como parâmetros para geração de uma superfície com limite quadrado (lado 400 $\mathrm{mm})$.

Nesta etapa, fica evidente a facilidade trazida pelo uso do gabarito, pois, ele traz as coordenadas $x$ e y (que são padronizadas), sendo necessário apenas anotar as medidas do eixo z (vertical). Assim, considerando o plano conhecido na porção superior da almofada, as posições de x e y são pré-estabelecidas no algoritmo visual por um slider de distância. Já as posições em z vão ser determinadas pela conversão das medidas na altura dos pontos da superfície.

A superfície pode ser exportada em formatos padrão CAD, ou ser convertida em malha de triângulos, como o formato STL, mais usual para fabricação digital. O modelo exportado pode ser utilizado para a usinagem de uma espuma, por exemplo.

Conforme apresentado na Figura 3, o algoritmo inicia de acordo com a quantidade de pontos oriundos das medições no eixo $z$, que é função das distâncias de $x$ e $y$ (Figura 3A). Esses dados numéricos podem ser adicionados pela importação de um arquivo no formato TXT (Figura 3B). Após, o algoritmo realiza o ordenamento dos dados, para corresponder ao posicionamento dos pontos no modelo paramétrico. Os pontos dão origem a curvas, as quais, por sua vez, geram uma superfície que, finalmente, é convertida em uma malha triangular para ser exportada no formato STL (Figura 3C). As etapas são explicadas mais detalhadamente a seguir.

Na Figura 3A é destacado o primeiro passo do algoritmo, o qual consiste, em parametrizar as medidas no plano xy (conforme o tampo do gabarito), considerando que, inicialmente, o eixo $z$ tem sempre o valor igual a zero. O controle do algoritmo pode ser realizado em função da resolução pretendida, ou seja, do número de pontos que se deseja medir em cada linha/coluna. Para tanto, devem-se configurar dois "Sliders", um para a distância e o outro para o número de pontos desejado. Assim, por meio do componente "Series", é criada uma lista de números com o intervalo definido. Na sequência, utilizou-se o componente "CrossRef", para que a lista de números anterior fosse 
replicada para os dois eixos (x e y), e, por fim, o componente "Point", o qual transforma essa lista em pontos no espaço.

Figura 3 - Algoritmo desenvolvido no editor visual Grasshopper. A) configuração dos pontos nos eixos x e y; B) leitura das medidas no eixo z;

C) geração de coordenadas em 3D, de superfície e de malha.

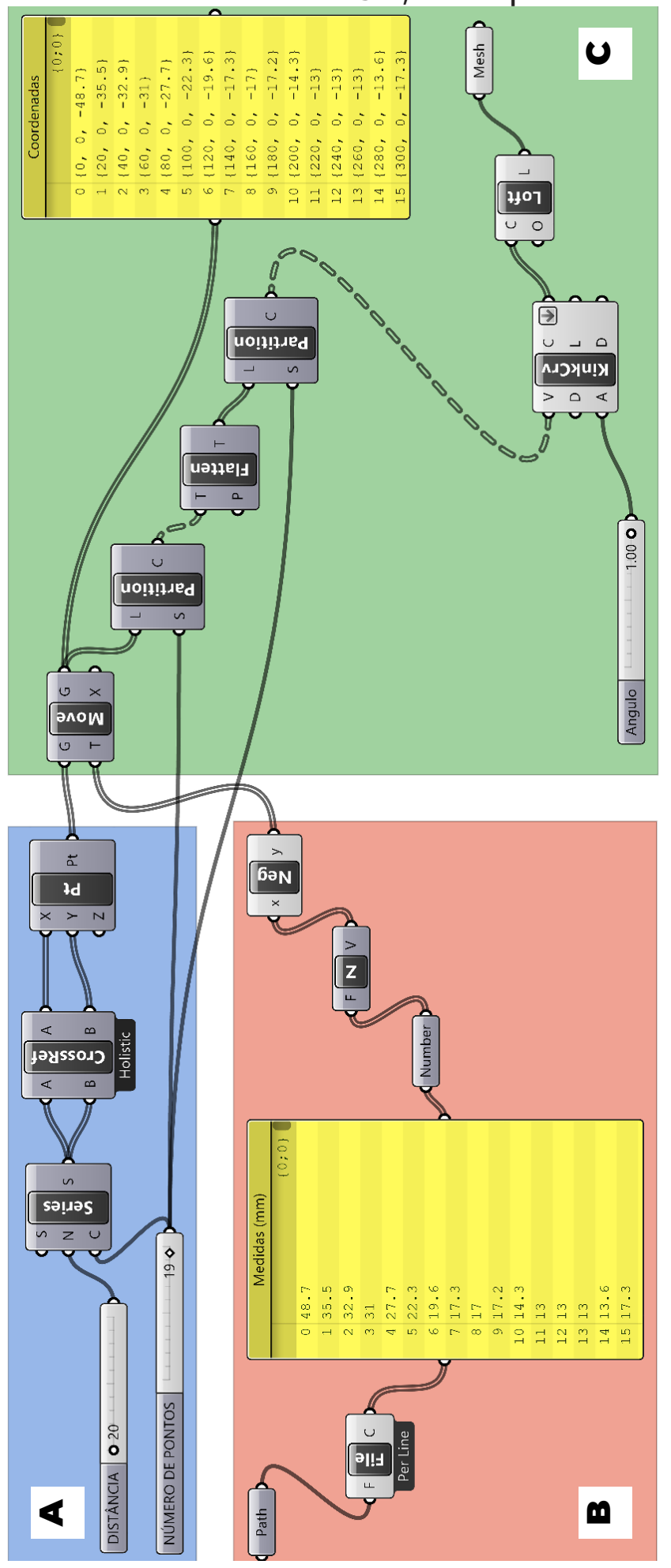

Fonte: Elaborado pelos autores, 2019. 
Na Figura 3B pode ser visualizada a segunda etapa do algoritmo, que consiste na importação do arquivo com os dados da medição e organização dos números, que darão origem às coordenadas z do modelo paramétrico. Inicialmente, por meio do "Path", se abre uma janela para inserção do caminho do arquivo a ser importado. A seguir, o componente "File" realiza a leitura do arquivo de texto, linha por linha, o que pode ser conferido no painel de medidas. Os dados do texto são transformados em números racionais ("Number") para permitir os cálculos do algoritmo. Então, os números são convertidos em um vetor ("Z") e transformados em negativos ("Neg"), visto que, as medidas partem da base superior do gabarito.

A terceira e última etapa do algoritmo pode ser observada na Figura 3C, nela os pontos são organizados em 3D e são realizadas operações para obter a malha final. A partir do componente "Move", os pontos referenciados em x e y são movidos em $z$, de acordo com as medidas definidas. A partir disso, tem-se uma nuvem de pontos ( $x, y$, z), a qual pode ser visualizada no painel de coordenadas. Embora com os pontos em questão seja possível gerar uma superfície, visando ter um maior controle de interpolação e suavização, optou-se por dividir as coordenadas, para gerar curvas (o que é feito pelos comandos "Partition", "Flatten" e "Partition" novamente). Os pontos referentes a cada curva são unidos com o componente "KinkCrv", que permite a inserção de um nó específico (kink) a partir de um ângulo limite (em radianos). No caso, foi definido o valor máximo de 1, para evitar a formação de segmentos de linha retos entre os pontos interpolados. Com as curvas suavizadas obtidas, o próximo passo é gerar uma superfície com o comando "Loft". Essa superfície pode ser usada em softwares CAD, ou, ainda, pode ser convertida em malha ("Mesh").

\subsection{Moldagem e execução}

Na porção interna do gabarito foi utilizada uma almofada moldável de posicionamento à vácuo, marca Versa Form, modelo 22" x 34" (56 x 86 cm). O modelo em questão é o mesmo utilizado no estudo de Prestes et al. (2012). Moraes (2009) descreve a almofada como sendo recheada com pequenas esferas de poliestireno e conectada a uma bomba de vácuo. Quando moldada, retira-se o ar de seu interior, unindo as esferas e mantendo a forma enquanto há vácuo.

Como pode ser observado na Figura 4A, a moldagem se dá quando o sujeito se senta sobre a almofada. Os cuidados com a adequação postural devem ser os mesmos sugeridos por Silva (2011), porém, utilizando o gabarito proposto, em vez de um escâner 3D para obter a forma. Neste caso, a almofada foi digitalizada apenas para servir de referência para posterior comparação.

Assim que a almofada adquire a forma (devido ao vácuo no interior), o sujeito pode sair e os dados de sua anatomia podem ser medidos manualmente com o auxílio do gabarito desenvolvido. Em cada ponto designado para medição, é inserida a haste de profundidade do paquímetro por três vezes, a fim de, poder trabalhar com uma média e minimizar a influência do operador nos erros metrológicos. A Figura 4B mostra a posição do paquímetro inserido em um dos pontos do tampo do gabarito, o qual, deve estar a $90^{\circ}$ e deve descer até encostar na almofada moldada. Deve-se tomar cuidado para a base do paquímetro encostar totalmente na face plana do gabarito, pois isso irá garantir o ângulo reto para a tomada da medida. 
Figura 4 - Coleta de dados: A) moldagem em almofada de posicionamento à vácuo; B) medição com paquímetro.
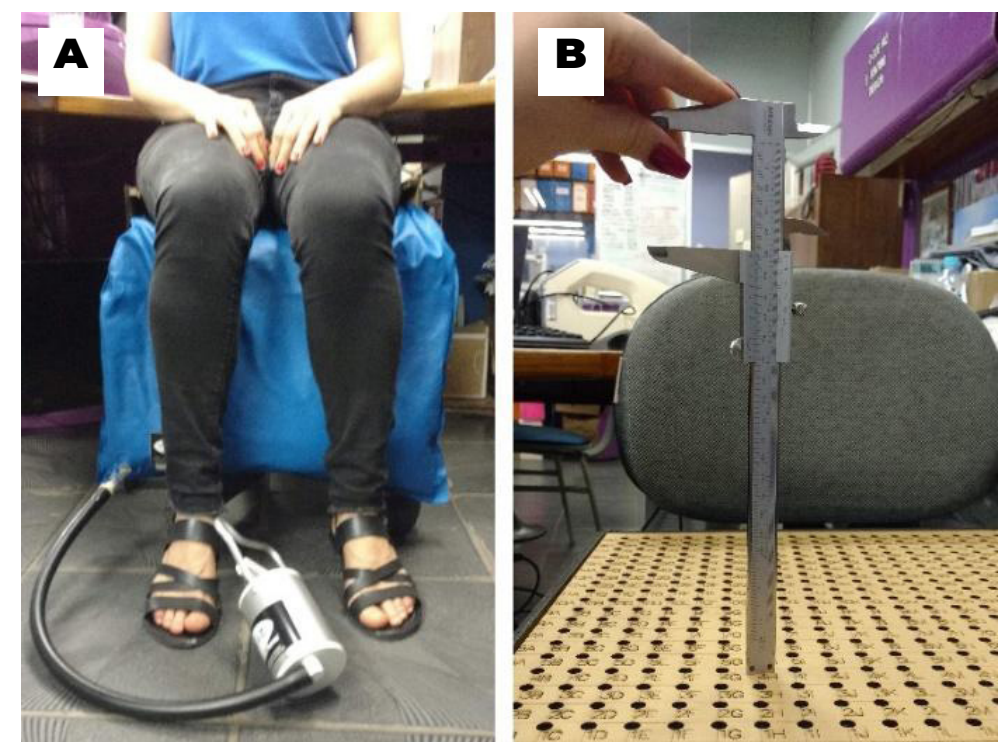

Fonte: Elaborado pelos autores, 2019.

A cada medição, o dado deve ser anotado em ordem combinada de linhas x colunas. Para facilitar o procedimento, criou-se uma tabela no Microsoft Excel. Cabe ressaltar que os dados podem ser anotados e transcritos em qualquer editor de texto, bastando colocar uma medida em cada linha (respeitando a ordem). Após a importação dos dados no algoritmo, tem-se o modelo gerado no software Rhinoceros, o qual foi convertido em malha e exportado para análise.

\subsection{Análise do modelo paramétrico}

Para a análise dimensional do modelo gerado pelo algoritmo proposto (Figura 5A), tomou-se como base a malha obtida por meio da digitalização 3D da almofada (figura 5B). O escâner 3D utilizado foi o Artec Eva, que se trata de um equipamento portátil, que opera com a tecnologia de luz branca estruturada. A digitalização foi realizada ainda com a almofada encaixada no gabarito, mas sem o tampo. Para ser usada como referência, foi obtida uma malha com resolução de 0,2 mm (Figura 5B).

As duas malhas obtidas, exportadas no formato STL, foram importadas no software Geomagic Qualify. Neste, foi realizado o alinhamento espacial dos modelos 3D (considerando os eixos $x, y$ e z), bem como, foi definido o modelo digitalizado como peça de referência e o modelo paramétrico como peça de teste, para fins de análise dimensional. 
Figura 5 - Modelos 3D: A) gerado pelo algoritmo desenvolvido; B) gerado por escâner 3D comercial.
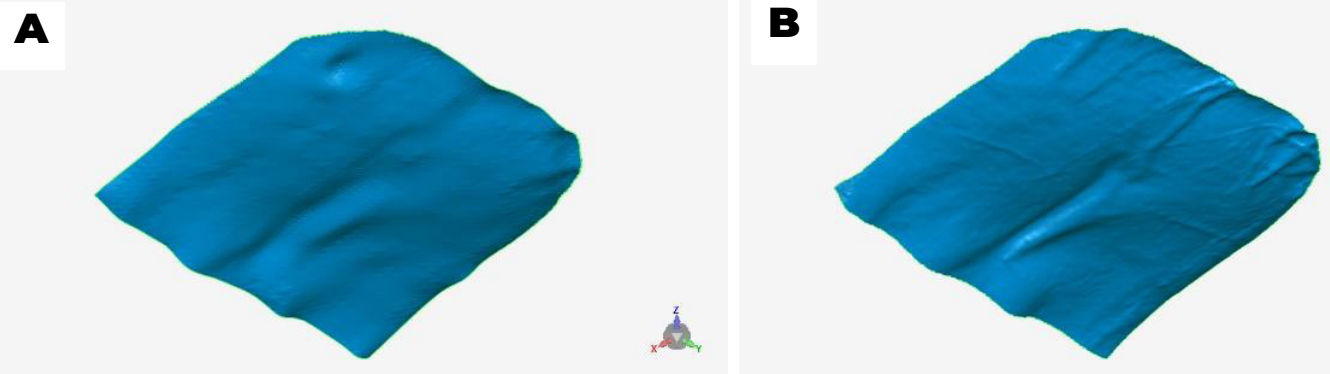

Fonte: Elaborado pelos autores, 2019.

Para o alinhamento dos modelos no espaço, esses foram sobrepostos com o uso da ferramenta Best Fit Alignment, a qual realiza iterações e calcula, o melhor encaixe fino entre eles. Quando a comparação 3D é realizada, pode ser determinado o maior desvio dimensional existente e ser estabelecido um espectro de cores que será incorporado aos modelos. $O$ espectro apresenta diferentes saturações entre o vermelho (erros em regiões onde o modelo está maior que a referência) e o azul (para regiões em que está menor). As regiões em que o desvio dimensional fica dentro de um valor limite estabelecido são representadas na cor verde. No presente estudo, o limite aceitável foi definido em 0,2 mm, que é a resolução do modelo gerado pelo escâner 3D.

Além do mapa de cores, o software fornece valores estatísticos dos pontos analisados, como média, desvio-padrão e desvio quadrático médio (RMS). O RMS, também chamado de valor eficaz, foi utilizado para a comparação, visto que ele é a raiz da média dos quadrados, e é um parâmetro mais sensível a valores dispersos.

\section{RESULTADOS E DISCUSSÃO}

O algoritmo possibilitou a geração de um modelo 3D de assento, quanto a isso, alguns pontos importantes são discutidos neste tópico. A principal questão, relacionada à moldagem, se refere às dobras formadas no tecido de revestimento da almofada de posicionamento a vácuo. Tais dobras podem ser observadas no modelo digitalizado com escâner 3D (Figura 5B).

Em relação ao modelo parametrizado pelo algoritmo, as dobras geram algumas distorções na malha resultante, como observado na Figura 6A. Isso ocorre devido à menor resolução, pois, assim, um ponto com altura muito diferente dos demais implica na deformação de uma região maior.

Para sanar esse problema, foi proposta uma filtragem de dados. O filtro consistiu em que cada medida fosse comparada aos seus oito pontos vizinhos. Assim, foi realizada a média das medidas e, se o valor do ponto era maior do que um limite (foi arbitrado $5 \mathrm{~mm}$ ), esse valor foi então substituído pela média calculada. Para exemplificar, hipoteticamente, se um ponto possui altura de $30 \mathrm{~mm}$, mas a média dos pontos vizinhos é de $10 \mathrm{~mm}$, sua altura foi alterada para esse segundo valor. Esse processo de filtragem possibilitou a suavização das interferências causadas pelas dobras do tecido da almofada, como pode ser percebido na Figura 6B. 
Figura 6 - Modelo parametrizado: A) gerado diretamente; B) com filtragem para suavizar o efeito de dobras.

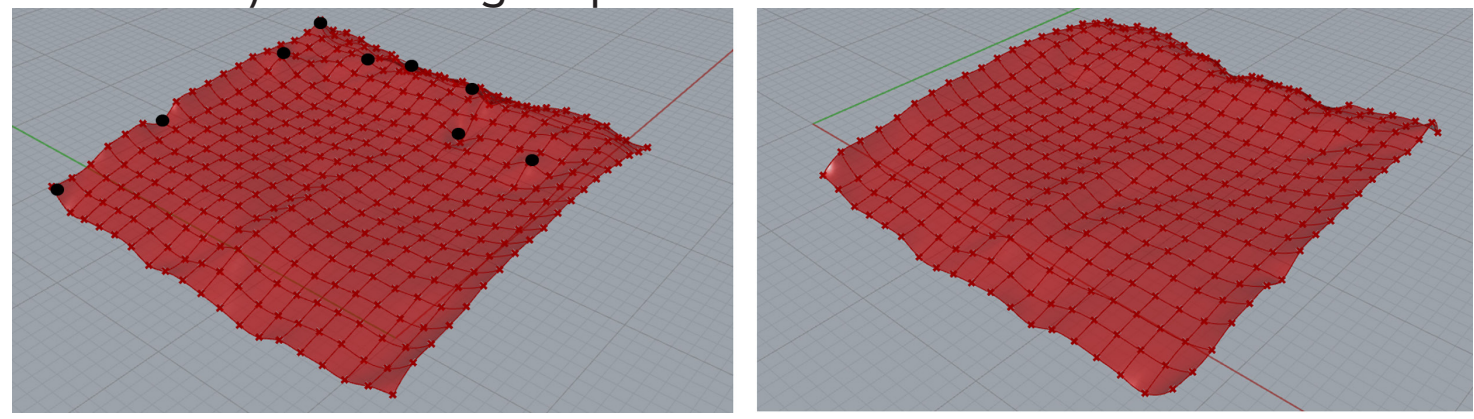

Fonte: Elaborado pelos autores, 2019.

Cabe ainda destacar que filtros podem ser adicionados ao algoritmo, mas também, podem ser aplicados com funções básicas no Microsoft Excel, facilitando algumas edições para pessoas não familiarizadas com programação. Essa questão das dobras também é observada por Silva (2011), pois, elas também constituem um problema nos modelos digitalizados e precisam ser tratadas. Nesse sentido, uma outra alternativa para minimizar esses efeitos é a utilização de fitas adesivas para cobrir as dobras antes da medição, o que pode evitar que a haste de profundidade do paquímetro adentre nas mesmas. Assim, fica a critério do utilizador atuar fisicamente no molde ou atuar digitalmente nos dados, conforme sua habilidade manual e/ou de programação.

Em relação a análise dimensional aplicada nos modelos, é obtido um valor RMS de 1,45 mm. A Figura 7 mostra o resultado da comparação entre o modelo paramétrico digital, com a aplicação do filtro de suavização, e o modelo digitalizado com escâner 3D. Nota-se que grande parte da porção de interesse está dentro das variações de azul-claro para amarelo-claro, verificado na escala como $\pm 1,4 \mathrm{~mm}$. Esse valor pode ser considerado satisfatório, baseado em Silva (2011), que considerou aceitáveis tolerâncias para moldagem manual de assentos de 3 a $4 \mathrm{~mm}$.

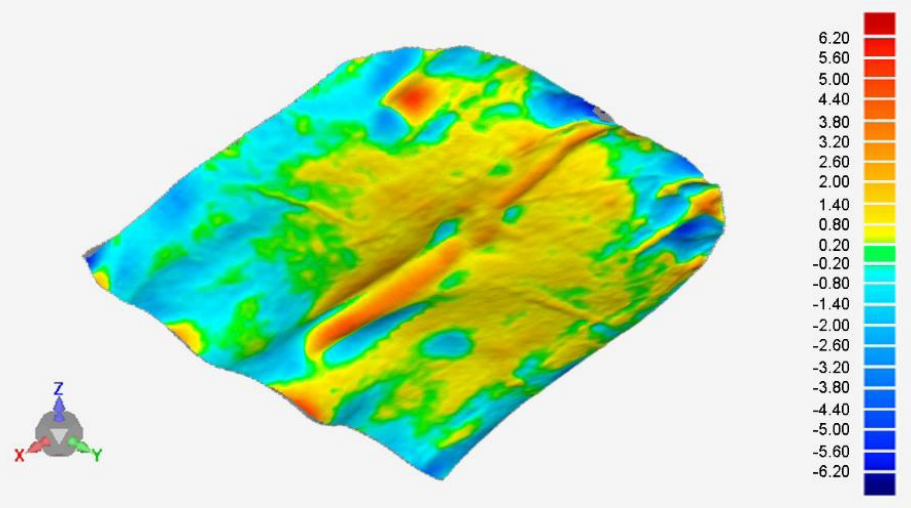

Figura 7 - Análise dimensional do modelo paramétrico obtido (escala em mm). Fonte: Elaborado pelos autores, 2019.

Ainda na Figura 7, é possível observar que há uma faixa mais alaranjada, com erros máximos próximos a $3,2 \mathrm{~mm}$, na região central do modelo, o que seria entre as pernas do usuário. Essa faixa se deve exatamente ao espaçamento ali existente, que faz com que se forme um sulco na almofada, o qual, precisaria de maior resolução para ser melhor representado. Essa é uma limitação do algoritmo, assim, considera-se que re- 
giões com contornos mais complexos podem não ser apropriadamente desenvolvidas com este método. De fato, dependendo do usuário de cadeira de rodas, conforme Silva (2011), é interessante projetar manualmente no local um cavalo de abdução, saliência que visa manter os membros inferiores abduzidos (joelhos afastados). Neste caso, visto que se trata apenas de um suporte, e não de uma parte do usuário a ser copiada, pode haver até mesmo a modelagem e inserção de um modelo externo. Por fim, é possível visualizar que as demais regiões de saturação em vermelho e azul (erros máximos) encontram-se nas bordas da área de interesse, algumas até nos limites da superfície, que podem até ser desconsideradas. Novos filtros podem ser criados para ajustar essas regiões, o que fica sugerido como trabalho futuro.

\section{CONCLUSÕES}

O método proposto, apesar de ainda poder ser otimizado, se mostra uma alternativa interessante para o desenvolvimento de assentos personalizados, em casos onde não se tem um escâner 3D disponível ou recursos para contratação do serviço de digitalização. Cabe destacar que o uso de almofadas de posicionamento é relativamente comum entre profissionais que realizam adequação postural. Assim, além de facilitar a moldagem, pode não ser necessário o investimento na compra deste equipamento.

A partir da análise do modelo paramétrico, verificou-se que um erro quadrático médio de 1,4 $\mathrm{mm}$ pode ser aceitável para um modelo de assento, especialmente considerando que a medição ocorre de forma manual. Quanto à região central do modelo, na qual, se identificaram erros de até $3,2 \mathrm{~mm}$, em alguns casos pode ser necessária uma intervenção na modelagem de um abdutor de pernas. Também pode ser utilizado um produto de mercado, porém, deve ser considerado o impacto no custo. Neste ponto, sugere-se a realização de estudos mais aprofundados quanto a questões econômicas, para que o método possa ser efetivamente considerado de baixo custo. Também são sugeridos como trabalhos futuros eventuais adaptações do método de geração do modelo, bem como, a otimização do algoritmo paramétrico e a criação de novos filtros para geração de modelos finais.

Considera-se que os resultados obtidos demonstram viabilidade do ponto de vista do desenvolvimento do protótipo inicial, teste, validação e sugestões de melhorias na proposta para aplicação do Design Paramétrico orientado para a Tecnologia Assistiva. Embora a parametrização digital possa ser considerada uma alternativa para obter a forma anatômica de um sujeito, é necessário ressaltar a necessidade da realização futura deste estudo, com uma amostragem significativa de pessoas com deficiência, para verificação de sua eficácia. Após a adequada avaliação de protótipos, pode-se partir para o desenvolvimento de um produto final. Para tanto, poderiam ser integrados conceitos de customização em massa à fabricação digital, por exemplo, padronizando um bloco de espuma a ser usinado de forma personalizada conforme a parametrização de cada usuário. 


\section{AGRADECIMENTOS}

Ao Conselho Nacional de Desenvolvimento Científico e Tecnológico (CNPq) e à Coordenação de Aperfeiçoamento de Pessoal de Nível Superior (CAPES) pelo apoio aos autores deste artigo.

\section{REFERÊNCIAS}

ASSOCIAÇÃO BRASILEIRA DE NORMAS TÉCNICAS. NBR ISO 7176-7: Cadeira de Rodas. Parte 7: Medição de dimensões de assentos e rodas. Rio de Janeiro: ABNT, 2009.

BERETTA, Elisa Marangon. Tecnologia Assistiva: Personalização em massa através do design e fabricação de assentos customizados para cadeiras de rodas. 2011. Dissertação (Mestrado em Design) - Programa de Pós-Graduação em Design, Universidade Federal do Rio Grande do Sul, Porto Alegre, 2011.

BERSCH, Rita de Cássia Reckziegel. Assistiva: Tecnologia e Educação. Disponível em: http://www.assistiva.com.br/tassistiva.html. Acesso em: 27 jul. 2018.

BERSCH, Rita de Cássia Recziegel et al. Fatores Humanos em TA: Uma Análise de Fatores Críticos nos Sistemas de Prestação de Serviços. Plurais - Revista Multidisciplinar, Salvador, v. 1, n. 1, p. 132-152, 2008.

BRASIL. Subsecretaria Nacional de Promoção dos Direitos da Pessoa com Deficiência. Comitê de Ajudas Técnicas. Tecnologia Assistiva. Brasília: CORDE, 2009. 138 p.

BRASIL. Ministério do Desenvolvimento, Indústria e Comércio Exterior. Instituto Nacional de Metrologia, Qualidade e Tecnologia. Programa de análise de produtos: relatório sobre a análise em cadeiras de rodas. Rio de Janeiro. 2013. Disponível em: http://www.inmetro.gov.br/consumidor/produtos/cadeira_r odas.pdf. Acesso em: 12 jul. 2018.

COSTA, Thays Neves et al. Uso da Digitalização 3D e da Parametrização de Medidas Antropométricas para Produção de Moldes Personalizados para o Vestuário. Revista Educação Gráfica, Bauru, v. 19, n. 2, p. 122-142, 2015.

CRYTZER, Theresa et al. Identifying characteristic back shapes from anatomical scans of wheelchair users to improve seating design. Medical Engineering and Physics, York, v. 38, n. 9, p. 999-1007, 2016.

DEMARCHI, Gustavo Scussel et al. O Desenho Paramétrico como facilitador na personalização de órteses para Tecnologia Assistiva. In: Sociedad Ibero-americana de Gráfica Digital, 21., 2017. Anais [...]. Chile, 2017. p. 22-24.

ENGSTRÖM, Bengt. Ergonomic Seating: A True Challenge. Germany: Posturalis Books, 2002. 
FERNANDES, Stefan von der Heyde; SILVA, Fabio Pinto; SILVA, Tânia Luisa Koltermann. Proposta de desenvolvimento de empunhadura personalizada para usuários com mal de parkinson. In: Congresso Brasileiro de Pesquisa e Desenvolvimento em Design, 11., 2014. Anais [...], Gramado 2014. p. 1-13.

FILHO, João Gomes. Ergonomia do objeto: sistema técnico de leitura ergonômica. São Paulo: Escrituras, 2003.

FLORIO, Wilson. Modelagem paramétrica, criatividade e projeto: duas experiências com estudantes de arquitetura. Gestão \& Tecnologia de Projetos, São Paulo, v. 6, n. 2, p. 43-66, 2012.

FREDRIKSSON, Peter; GADDE, Lars-Erick. Flexibility and rigidity in customization and build-to-order production. Industrial Marketing Management, Holanda, n. 34, p. 695705, 2005.

GIL, Antonio Carlos. Métodos e técnicas de pesquisa social. 6. ed. São Paulo: Atlas, 2008.

JONES, Peter R. M.; RIOUXB, Marc. Three-dimensional Surface Anthropometry: Applications to the Human Body. Optics and Lasers in Engineering, Inglaterra, n. 28, p. 89-117, 1997.

KRÜGER, Jaqueline Menezes; FERREIRA, Alexandre Rodrigues. Aplicação da Tecnologia Assistiva para o Desenvolvimento de uma Classe Ajustável para Cadeirantes. Iberoamerican Journal of Industrial Engineering, Florianópolis, v. 5, n. 9, p. 43-69, 2013.

MORAES, Helton Scheer de. Projeto Conceitual de Sistemas de Assento para cadeira de rodas: Uma Abordagem Sistemática. 2009. Dissertação (Mestrado em Design) Programa de Pós-graduação em Design, Universidade Federal do Rio Grande do Sul, Porto Alegre, 2009.

OMS - ORGANIZAÇÃO MUNDIAL DA SAÚDE. Secretaria de Estado dos Direitos da Pessoa com Deficiência. Wheelchair ServiceTraining Package:Basic Level. São Paulo,2014.88 p. Disponível em: https://apps.who.int/iris/bitstream/handle/10665/78236/9789241503471_ reference_manual_por.pdf?sequence=48. Acesso em: 25 jul. 2018.

OXMAN, Rivka; OXMAN, Robert. Theories of the digital in architecture. Nova Yorque: Routledge, 2014.

PIRES, Janice de Freitas; PEREIRA, Alice Theresinha Cybis; GONÇALVES, Alexandre. Taxonomias de Geometria da Arquitetura Contemporânea: Uma Abordagem Didática ao Ensino da Modelagem Paramétrica na Arquitetura. Gestão \& Tecnologia de Projetos, São Paulo, v. 12, n. 3, p. 27-46, 2017.

PONTES, Fernando Vicente de. Adequação postural em cadeiras de rodas de pessoas 
com deficiência: estudo retrospectivo. 2017. Dissertação (Mestrado em Ciências) - Programa de Ciências da Reabilitação, Universidade de São Paulo, São Paulo, 2017.

PRESTES, Rafael Cavalli et al. Development and Thermographic Analysis of Custom Seats for Wheelchairs. In: BREEDON, Philip (org.). Smart Design. Londres: Springer London, 2012. p. 97-102.

PRODANOV, Cleber Cristiano. Metodologia do trabalho científico [recurso eletrônico]: métodos e técnicas da pesquisa e do trabalho acadêmico. 2. ed. Novo Hamburgo: Feevale, 2013.

RENNER, Jacinta Sidegum et al. Tecnologias Assistivas e Cadeira de Rodas Infantil: uma discussão com enfoque para o conforto, os aspectos lúdicos e a inclusão social. Revista Observatório, Palmas, v. 4, n. 3, p. 156-179, 2018.

SILVA, Fabio Pinto da. O uso da Digitalização Tridimensional a Laser no Desenvolvimento e Caracterização de Texturas Aplicadas ao Design de Produtos. 2006. Dissertação (Mestrado em Engenharia) - Programa de Pós-Graduação em Engenharia de Minas e Metalúrgica e Materiais, Universidade Federal do Rio Grande do Sul, Porto Alegre, 2006.

SILVA, Fabio Pinto da. Usinagem de espumas de poliuretano e digitalização tridimensional para fabricação de assentos personalizados para pessoas com deficiência. 2011. Tese (Doutorado em Engenharia) - Programa de Pós-Graduação em Engenharia de Minas e Metalúrgica e Materiais, Universidade Federal do Rio Grande do Sul, Porto Alegre, 2011.

TEDESCHI, Arturo. AAD_Algorithms - Aided Design: Parametric strategies using Grasshopper. Itália: Le Penseur, 2014.

TEIXEIRA, Érika et al. Terapia ocupacional na reabilitação física. São Paulo: Roca, 2003. 